Meta

Journal des traducteurs

Translators' Journal

\title{
Les hybrides lexicaux en français contemporain : délimitation du concept
}

\section{Jan Kortas}

Volume 54, numéro 3, septembre 2009

URI : https://id.erudit.org/iderudit/038313ar

DOI : https://doi.org/10.7202/038313ar

Aller au sommaire du numéro

\section{Éditeur(s)}

Les Presses de l'Université de Montréal

ISSN

0026-0452 (imprimé)

1492-1421 (numérique)

Découvrir la revue

Citer cet article

Kortas, J. (2009). Les hybrides lexicaux en français contemporain : délimitation du concept. Meta, 54(3), 533-550. https://doi.org/10.7202/038313ar
Résumé de l'article

Le présent article a pour but de délimiter le concept d'hybride lexical en français contemporain et d'en proposer une typologie en fonction de différents critères. L'hybride lexical est défini comme un néologisme issu principalement d’une hybridation, considérée comme un processus spécifique de créativité lexicale, qui combine les mécanismes de dérivation et d'emprunt direct. Ce processus se réalise par l'adjonction d'un élément allogène (mot plein lié, confixe ou affixe), emprunté directement à une langue $\mathrm{A}$, soit à une base indigène, soit à un élément allogène venant directement d'une langue B. Le français emprunte aussi des hybrides à d'autres langues dans lesquelles ils sont formés en vertu de mécanismes hybridogènes analogues. Les formants d'hybrides se caractérisent par une absence d'autonomie dans la langue emprunteuse. Selon divers paramètres, les hybrides lexicaux peuvent se diviser en : 1) hybrides dérivationnels et hybrides par emprunt ;2) hybrides interférentiels (résultat d'une interférence de deux langues dans des régions limitrophes) et hybrides non interférentiels ; 3) hybrides dénominatifs et hybrides d'auteur à fonction poétique et ludique.
Ce document est protégé par la loi sur le droit d'auteur. L'utilisation des services d'Érudit (y compris la reproduction) est assujettie à sa politique d'utilisation que vous pouvez consulter en ligne.

https://apropos.erudit.org/fr/usagers/politique-dutilisation/ 


\title{
Les hybrides lexicaux en français contemporain : délimitation du concept
}

\author{
JAN KORTAS \\ Université de Gdańsk, Gdańsk, Pologne \\ jan.kortas@univ.gda.pl
}

\begin{abstract}
RÉSUMÉ
Le présent article a pour but de délimiter le concept d'hybride lexical en français contemporain et d'en proposer une typologie en fonction de différents critères. L'hybride lexical est défini comme un néologisme issu principalement d'une hybridation, considérée comme un processus spécifique de créativité lexicale, qui combine les mécanismes de dérivation et d'emprunt direct. Ce processus se réalise par l'adjonction d'un élément allogène (mot plein lié, confixe ou affixe), emprunté directement à une langue $A$, soit à une base indigène, soit à un élément allogène venant directement d'une langue $B$. Le français emprunte aussi des hybrides à d'autres langues dans lesquelles ils sont formés en vertu de mécanismes hybridogènes analogues. Les formants d'hybrides se caractérisent par une absence d'autonomie dans la langue emprunteuse. Selon divers paramètres, les hybrides lexicaux peuvent se diviser en: 1) hybrides dérivationnels et hybrides par emprunt; 2) hybrides interférentiels (résultat d'une interférence de deux langues dans des régions limitrophes) et hybrides non interférentiels; 3) hybrides dénominatifs et hybrides d'auteur à fonction poétique et ludique.
\end{abstract}

\begin{abstract}
The aim of the paper is to define the categorial status of a lexical hybrid in modern French and to classify hybrids according to various typological criteria. A lexical hybrid has been defined as a neological type arising mostly as a result of hybridization, thus a specific derivational process being a combination of derivation and direct borrowing, and depending on the addition of a foreign-language formative (lexeme, confix, affix) borrowed directly from language $A$ either to the native base or to a foreign element borrowed directly from language B. The French language has borrowed some hybrids from foreign languages, in which these formations arose as a result of analogous hybridogenic processes. Hybrid formatives are characterized by a lack of autonomy in the borrowing language. The division of hybrids is presented as follows: 1) derivational hybrids and hybrid-borrowings; 2 ) interferential hybrids (created as a result of two languages affecting each other because of their geographical proximity) and non interferential hybrids; 3) nominative hybrids and authorial hybrids (the latter, as nonce-words, are created for the aims of poetry, satire and popular entertainment).
\end{abstract}

\section{MOTS-CLÉS/KEYWORDS}

néologie, dérivation, emprunt, hybride lexical, confixe neology, derivation, borrowing, lexical hybrid, confix 


\section{Introduction}

La néologie hybride contribue aujourd'hui à enrichir le lexique de différents registres de langue, surtout celui des technolectes (anticorps, ovocyte), des médias et de la publicité (pancake), ainsi que celui de certains sociolectes, par exemple, le langage des jeunes ou le français des cités (top-niveau, couillav). Cependant, dans les travaux portant sur la lexicologie et la dérivation, les concepts d'hybride et d'hybridation sont flous, voire équivoques: les tentatives d'analyse et de classement, fort fragmentaires, se limitent généralement à étudier le type structurel classique d'hybride en français (hybride confixal), qui se répand dans cette langue depuis le XVIII siècle avec l'expansion de la terminologie scientifique et technique (aéroduc: hybride gréco-latin). Étant trop attachés à la conception traditionnelle de ce concept, les linguistes francophones prennent rarement en considération le caractère dynamique et changeant du phénomène d'hybridation, d'où l'absence d'une théorie de la néologie hybride qui soit cohérente. Par contre, le processus d'hybridation est étudié plus en profondeur par les linguistes slaves, surtout polonais et tchèques (Buttler 1986, 1990; Obara 1986; Warchoł 1986). Il existe d'ailleurs des divergences de nature étymologique entre, d'une part, le polonais ou le tchèque et, d'autre part, le français, divergences qui justifient partiellement ce décalage méthodologique. En effet, le caractère hybride de certaines formations suffixales slaves est mieux visible à cause d'une nette différence étymologique entre leurs éléments indigènes (slaves) et allogènes (par exemple, des suffixes d'origine latine). Par contre, étant donné la parenté historique du français et du latin, le contraste entre la base lexicale indigène (française) et le suffixe allogène (latin) est souvent effacé, d'où une difficulté accrue de déceler des structures hybrides.

Qui plus est, en linguistique, les termes hybride et hybridation dépassent le cadre traditionnel de la morphologie et du lexique, ce qui est dû au sémantisme générique de la notion même (de hibrida en latin: /de sang mêlé/; terme utilisé d'abord dans les sciences biologiques, pour désigner un individu provenant du croisement de variétés, d'espèces différentes, puis dans l'acception courante: /composé d'éléments de nature différente, anormalement réunis/). Ce sémantisme invite donc à proposer les acceptions linguistiques les plus variées de la notion en question. Nous signalerons ce problème dans la section 2 .

L'étude des hybrides sur le plan morpho-lexical devrait avoir surtout pour but de préciser des critères stricts qui permettent de délimiter cette catégorie et de la distinguer de formations lexicales apparentées. Les auteurs confondent ou identifient souvent deux types d'unités lexicales: les hybrides et les emprunts assimilés sur le plan dérivationnel. Certes, la structure hybride, elle aussi, est un type particulier d'emprunt, mais, à notre avis, les deux catégories reposent sur des mécanismes dérivationnels différents, et, par conséquent, elles devraient être distinguées l'une de l'autre.

La délimitation de l'hybride sur le plan lexical pourrait servir de point de départ pour proposer une typologie des hybrides suivant des critères variés, comme les mécanismes de naissance, la structure morphologique, les fonctions néologiques, etc. D'amples perspectives d'étude de ce concept s'ouvrent aussi sous les angles sociolinguistique, statistique, normatif, diachronique, etc. (types de productivité hybride à travers le temps, types de sociolectes caractérisés par une fréquence accrue des structures hybrides, productivité de l'hybridation gréco-latine et anglo-américaine aujourd'hui, néologie hybride du point de vue normatif, etc.). 
Le présent article a pour objet la délimitation du concept d'hybride lexical et une proposition de typologie de formations hybrides selon différents critères.

\section{Différentes acceptions des termes hybride et hybridation en linguistique}

Parmi les différentes acceptions non lexicales de ces termes, mentionnées par les linguistes francophones, notons surtout:

a) L'hybridation comme pléonasme syntaxique

Certains auteurs (Berrendonner 1997: 82) appellent hybridation une structure syntaxique fautive, de type pléonastique, qui résulte de l'adjonction de deux structures rectionnelles distinctes (par ex., quelqu'un à qui on lui pose la question).

b) L'hybridation comme interférence linguistique fautive

En étudiant les fautes d'interférence commises par les Franco-Ontariens, Forlot (1999) appelle hybride ou emprunt mixte toutes sortes d'interférences fautives entre les structures françaises et anglaises:

- niveau dérivationnel: une certaine nobilité ${ }^{1}$; du temps de la royalté(croisement de nobility, royalty et du morphème français -é);

- niveau morpho-syntaxique: je serais contenté d'avoir... (croisement de je me serais contenté de... et de I would be contented with...); elle est pas vraiment fluente (adjonction d'une marque du féminin à l'adjectif anglais fluent, ayant le sens de /qui parle couramment/).

c) La langue hybride comme langue mixte

Au sens général, la langue hybride désigne un parler qui emprunte certains traits à deux ou plusieurs langues. Certains auteurs rétrécissent cette acception courante, en appelant langue hybride un parler constitué d’un mélange de codes, qui résulte du contact des langues mais non du besoin d'intercompréhension, ce dernier cas étant celui du pidgin, par exemple (Hamers et Blanc 1983: 255).

En poursuivant cette approche générale qui s’inscrit en dehors de la morphologie lexicale, on pourrait voir des phénomènes hybrides aussi à d'autres niveaux de la langue, où l'on a affaire à une concurrence d'éléments qui viennent de langues différentes. Ainsi, l'emprunt top secret peut être considéré comme un hybride phonétique, vu la prononciation anglaise du premier élément, et la prononciation francisée du second. Certains mots grammaticaux résultent, eux aussi, du croisement d'éléments venant de langues différentes. Dans cette optique, cadhuna (de kata, préposition grecque, $+\underline{\text { unam }}$, numéral latin), un adjectif indéfini attesté dans les Serments de Strasbourg (842), peut être considéré comme une première formation de type hybride attestée dans le texte écrit français. C'est aussi sur le plan de la syntagmatique phrastique que l'on rencontre des structures hybrides («renvoyer une affaire sine die»). Encore une fois, ce sont surtout les linguistes slaves (voir plus haut) qui étudient des structures et des phénomènes hybrides sur le plan phonologique, flexionnel, syntaxique, idiomatique, stylistique, textuel ou dialectologique.

\section{Hybride lexical : état de la question}

Le terme hybride dans son acception linguistique a été utilisé pour la première fois par Vaugelas (1647/1981: 273), qui, en soumettant à l'examen normatif les expressions au préalable, préalablement, fait la remarque suivante: «[...] ils [ces mots] avaient 
quelque chose de monstrueux en ce qu'ils étaient moitié latins et moitié français, quoiqu'en toutes les langues il y ait beaucoup de mots hybrides [...] ou métis [...]».

Le concept d'hybride lexical est lié en premier lieu à l'apparition en français des formations du type messidor, qui sont une altération de la composition savante (par exemple, nécropole), dite aussi recomposition classique (Gaudin et Guespin 2000). Celle-ci, en vogue surtout depuis le XviII ${ }^{\mathrm{e}}$ siècle, consiste en la jonction de deux éléments tirés directement du grec ou du latin et appelés, selon les linguistes, confixes, quasimorphèmes, pseudomorphèmes ou paléomorphèmes. Comme l'a fait remarquer Gross (1996), le fonctionnement de ces éléments se situe entre celui des affixes et celui des mots autonomes (ils ont une fonction lexicale, comme des noms, des adjectifs ou des verbes, mais, à l'instar des affixes, ils sont dépourvus d'autonomie dans la langue emprunteuse). L'altération du composé savant prend la forme d'un mot comportant deux confixes tirés chacun de deux langues différentes, en l'occurrence le latin (messi-) et le grec (-dor). Ce type de structure a donné naissance à une définition traditionnelle de l'hybride lexical, que l'on peut trouver, par exemple, dans le Dictionnaire de linguistique: « un mot composé dont les constituants sont empruntés à des racines de langues différentes» (Dubois et al. 1973: 246). Or, la définition de Dubois a aujourd'hui l'inconvénient d'être trop restreinte, car elle limite le concept en question à des formations qui ne se composent que de racines, donc d'éléments lexématiques, ce qui implique surtout la présence de confixes gréco-latins (aéroduc).

Le premier classement d'hybrides, proposé par Darmesteter et Hatzfeld (1964), regroupe dans le cadre de la composition savante, les types suivants:

- hybrides gréco-latins (autoclave, chloral)

- hybrides gréco-français (bureaucratie, antipape)

- hybrides franco-latins (génito-urinaire, séro-sanguin)

La classification des auteurs, fondée essentiellement sur des critères étymologiques (éléments grecs, latins et français), ne tient compte ni des différences structurelles qui résultent de la dichotomie synchronie / diachronie, ni de la typologie morphologique (mélange de différents types morphématiques d'hybrides à l'intérieur d'une même catégorie étymologique).

D'autres auteurs suivent cette conception classique, fondée principalement sur le mécanisme de confixation. Voici un classement d'hybrides fait par Guilbert (1975):

- élément grec + élément latin (aéroduc, ptéronave)

- élément latin + élément grec (spectroscope, altimètre)

- élément grec + nom français ayant une autonomie lexicale (auto-mouvoir, hydronef)

- élément grec ou latin + élément composé (aéro-biplane, semi-orthoptère)

Une classification semblable, basée sur un amalgame d'éléments grecs, latins et français a été proposée par Leclercq $(1976,1989)$. La principale différence par rapport à celle de Guilbert réside dans le fait que l'auteur y inclut aussi des formations suffixales, en donnant comme exemples des néologismes nés sous la Révolution: monarchien ou aérostier (/celui qui manœuvre un aérostat/).

La première classification d'hybrides, qui dépasse le cadre traditionnel de la confixation, et qui est en même temps la plus synthétique, a été élaborée par Galliot (1954). Faute de place, nous la présentons dans ses grandes lignes: 
- hybrides gréco-latins (Technicalor, Audios)

- français et langues anciennes (monocoque, Vita-raisin)

- hybrides à éléments anglais (Téléfast, Vitahair)

- hybrides à élément chimique (Iodorganine, Théosalvose)

Malgré de nombreux inconvénients (classement peu cohérent combinant des critères étymologiques et taxinomiques, terminologie ambiguë et partiellement désuète), la classification de Galliot a des avantages incontestables: elle prend surtout en considération des éléments allogènes autres que ceux d'origine gréco-latine, notamment anglo-américains, en montrant leurs différents statuts morphématiques et possibilités combinatoires (toutes sortes d'amalgames de confixes traditionnels, mots gréco-latins, éléments français et anglo-américains). Par sa richesse structurelle et sa variété d'exemples, l'étude de Galliot annonce une vague de néologismes hybrides typiques de l'époque médiatique (langage publicitaire, journalistique, etc.).

Différents auteurs d'ouvrages récents classent dans les hybrides les productions néologiques les plus variées, admettant de multiples croisements d'éléments grécolatins et anglo-américains:

- nom + nom: photo finish ${ }^{2}$

- synapsie: toiture à sheds;

- adjectif + nom: big baffe;

- confixe + nom: philonazi;

- nom + confixe: clochophobe;

- confixe + confixe: somnogène;

- préfixe (allogène ou indigène) + nom: hypercorrection, surbooking;

- nom + suffixe (allogène ou indigène): ramping, kitchenette;

- lexies complexes: self-made-mélomane, hypofifriophobie.

Certains linguistes (Beciri 1994; Cabré 1998) conçoivent l'hybride lexical de manière toute nouvelle, suivant le principe de néologicité. Il ne s'agit plus d'une hétérogénéité d'éléments basée notamment sur l'opposition grec / latin (amalgame de confixes grec et latin dans une lexie). En effet, de telles formations sont aujourd'hui considérées comme régulières et normativisées, donc elles n’ont rien d'hybride. Selon ces auteurs, les lexies hybrides ne seraient que des productions néologiques récentes, souvent de type fantaisiste, caractérisées par l'opposition base indigène / formant allogène. En revanche, les formations traditionnelles de type gréco-latin, comme pluviomètre, ne seraient pas des hybrides, contrairement à accidentogène, humidologie ou cinéphile.

Dans ce panorama des différentes acceptions de l'hybride lexical chez les auteurs francophones, on peut distinguer deux tendances principales:

1) L’approche classique, qui repose essentiellement sur le mécanisme de confixation (totale ou partielle). Malgré ce dénominateur commun qu'est le confixe, le concept traditionnel d'hybride a des acceptions toutes différentes, suivant l'auteur. Une conception sensu largo englobe, outre les types traditionnels des hybrides confixaux (télévision), des formations apparentées, comme les recomposés modernes (téléspectateur) ou les mots-valises (internaute $=$ internet + astronaute).

2) L’approche contemporaine, qui élargit les contours du concept en question en dehors de son extension confixale. Les auteurs distinguent les néologismes hybrides morphologiquement les plus variés (amalgames d'éléments lexématiques, affixaux et 
désinentiels), qui admettent de multiples croisements étymologiques. Cependant, ils ne se servent du terme hybride qu'à titre fortuit, en étudiant d'autres problèmes lexicologiques, comme la néologie (Sablayrolles 2000), les emprunts, les calques, les langues de spécialité, etc. (Trescases 1983; Nicolas 1994; Quérin 2001; Galliot 1954).

\section{Hybride lexical : délimitation du concept}

Contrairement à la plupart des linguistes qui étudient les hybrides lexicaux sur le plan de la morphologie dérivationnelle, avec ses trois grands types de mécanismes néologiques (composition, recomposition [dérivation savante] et affixation [ReyDebove 1984]), nous proposons d'élargir les contours catégoriels de l'hybride lexical en dehors de ce cadre classique. Pour ce faire, nous nous servirons du concept de lexie, bien implanté en lexicologie récente, élaboré notamment par Pottier et Sablayrolles. De fait, certains auteurs étudiant le phénomène d'hybridation lexicale dépassent ce cadre morphématique traditionnel. Ainsi, Humbley (1974) parle de lexies complexes hybrides (après spray crème) et Depecker (2000) de syntagmes hybrides (circuit full custom).

Parmi les types de lexies distingués par Sablayrolles (2000), nous reprenons ceux qui s'adaptent le mieux à l'étude des hybrides: lexies construites (correspondant grosso modo aux trois types traditionnels de la morphologie dérivationnelle selon Rey-Debove (1984), syntagmes lexicalisés ou synapsies et expressions ou locutions. En effet, si l'on considère des synapsies comme harpon de spudding, navire à welldeck, ou des locutions telles que grosso merdo, être croque-love, on s'aperçoit que leur caractère hybride est manifeste. Les hybrides synaptiques sont surtout caractéristiques du langage scientifique et technique, et les hybrides locutionnels s'observent en premier lieu en français familier, notamment dans sa variante branchée.

\subsection{Critères d'hybridité}

Faute de méthode cohérente qui permettrait de délimiter les hybrides lexicaux, les auteurs conçoivent ce concept, comme nous l'avons vu plus haut, de manière différente, en se basant sur des critères différents et en y appliquant les combinaisons morphématiques les plus variées. Nous essayerons de délimiter une catégorie d'hybride lexical transgressant son acception classique généralement reconnue (de type confixal) au moyen d'une série de critères pertinents (les critères «subjectivisants » de type normatif ou néologique seront d'une importance secondaire), qui permettront surtout d'éviter une confusion entre hybride et catégories apparentées, comme les emprunts assimilés au niveau dérivationnel.

\subsubsection{Principe synchronique de délimitation des hybrides lexicaux}

Les paramètres synchroniques s'appliquent automatiquement dès que l'on cherche à établir un statut de l'hybride en tant que type dérivationnel en français contemporain. La confusion des deux critères dans la description des hybrides, synchronique et diachronique, comme c'est le cas chez Guiraud (1968) ou chez Hamon (1992), par exemple, a deux conséquences: premièrement, le concept d'hybride se dilue, englobant aussi certains mots complexes non construits, comme cauchemar (considéré par Hamon comme un hybride comportant un élément roman et un élément germanique); deuxiè- 
mement, certains hybrides vrais du point de vue synchronique sont classés suivant des critères purement étymologiques (d'après Hamon: bureaucratie - un hybride gréco-latin où cette historicité abusive affecte l'élément français bureau).

L'approche diachronique dans l'étude des formations hybrides est particulièrement visible en onomastique (Hamon 1992; Nègre 1990), car beaucoup d'anthroponymes et de toponymes, nés il y a des siècles dans des zones linguistiquement limitrophes, présentent des amalgames d'éléments étymologiquement différents, entièrement lexicalisés aujourd'hui.

L'importance de la méthode synchronique dans l'étude des phénomènes hybrides est mise en relief par des lexicologues polonais, comme Buttler (1986, 1990), Obara (1986) et Warchoł (1986). Selon ce dernier, pour conférer à une lexie un statut d'hybride, il convient surtout de préciser si en synchronie, c'est-à-dire au moment de la formation de cette unité, un élément lexématique venant d'une langue étrangère est non autonome dans la langue emprunteuse, contrairement à son statut autonome dans la langue prêteuse. Si cette condition est remplie, cet élément acquiert l'étiquette d'allogène, l'allogénéité d'un formant étant un des paramètres essentiels de l'hybridité (voir plus bas).

$\mathrm{Si}$, dans la formation des hybrides, le principal paramètre d'allogénéité pour un élément lexématique est sa non-autonomie dans la langue emprunteuse, pour un élément grammatical (notamment suffixal), il consiste en l'emprunt direct, en synchronie, de cet élément à une langue étrangère.

En vertu de ces critères de synchronie, un composé comme homme-sandwich ne serait pas un hybride, car l'élément sandwich, quoique d'origine anglaise, n'est qu'un emprunt en diachronie. En effet, au moment de la formation de cette lexie, cet élément préexistait en français comme mot autonome, bien assimilé dans cette langue. Par contre, le composé médecine-ball serait considéré comme un hybride, l'élément ball, emprunté directement à l'anglais, étant privé d'autonomie en français.

Pour illustrer la suffixation, le nom vieillard, malgré le suffixe -ard d'origine germanique, n'est pas un hybride en français contemporain, car l'élément germanique hart ajouté aux bases françaises a subi, à travers les siècles, une francisation complète (une assimilation formelle, et notamment une grammaticalisation très poussée).

Néanmoins, nous sommes loin d'exclure totalement, dans l'étude des hybrides, l'utilité de l'approche diachronique. Celle-ci aide à distinguer les différents facteurs de l'hybridation, c'est-à-dire du mécanisme dérivationnel qui soude des éléments originairement hétérogènes, comme le bilinguisme (qui fait naître surtout des hybrides anthroponymiques et toponymiques, par exemple Nashville, Greenville dans l'ancienne Louisiane) ou l'analogie (automobile d'après locomobile).

L'optique diachronique contribue aussi à préciser le degré d'allogénéité de certains éléments, par exemple de suffixes de type savant (voir plus bas). Elle permet également d'étudier la productivité des différents types d'hybridation à une époque donnée, d'analyser le rôle des facteurs historiques et géographiques qui accompagnent la naissance des formations hybrides, etc. Finalement, elle aide à délimiter certains éléments confixaux gréco-latins difficiles à déchiffrer sans savoir historique. Nous développons cette problématique dans ce qui suit. 


\subsubsection{Degré de compétence linguistique}

Nous reprenons là une question posée par Buttler (1990): quel type de compétence doit-on prendre en considération pour évaluer le caractère hybride d'une lexie? Celle du sujet parlant moyen ou bien celle du linguiste, compte tenu de son savoir théorique, et en particulier historique? Le même problème, quoique dans une perspective plus générale, car concernant la place des informations étymologiques dans la morphologie synchronique, est soulevé par Corbin:

Le linguiste se trouve partagé entre les deux hypothèses suivantes:

- Une hypothèse minimale, qui considérera comme dérivationnelles des relations perçues comme telles par un locuteur moyen n’ayant pas nécessairement appris le latin et/ou le grec, et qui rejettera toute relation extérieure à ces relations minimales communes.

- Une hypothèse maximale, qui inclura dans les relations dérivationnelles toutes les relations étymologiquement motivées, aussi bien formellement que sémantiquement. (Corbin 1987: 96)

D’après Corbin (1987: 101), l'étymologie et, plus largement, les informations historiques, permettent: a) d'élargir le champ des données synchroniques «pour expliquer le comportement linguistique synchronique des mots construits, quand tous les autres types d'explication se sont révélés inopérants [...]»; b) de compléter la compétence défaillante du morphologue synchronicien.

Comme les hybrides sont par définition des lexies qui combinent des éléments venant de langues différentes, l'hypothèse maximale, telle qu'elle est envisagée par Corbin, semble plus adéquate, surtout en ce qui concerne les hybrides confixaux gréco-latins. Ceux-ci, souvent indivisibles morphologiquement pour le locuteur moyen, notamment dans le cas de différents technolectes (en médecine, par exemple), présentent toutefois une structure morphématique propre aux mots construits, analysable suivant les mécanismes synchroniques auxquels peuvent s'ajouter des outils d'analyse de type diachronique.

\subsubsection{Hybride lexical - résultat du procédé dérivationnel d'hybridation}

Nous employons ici les termes dérivation et dérivationnel au sens large du mot pour désigner, de manière générale, la formation de nouvelles lexies.

Dans l'optique dérivationnelle, une question fondamentale se pose: quelle est la place, dans la formation des hybrides, d'une part, des mécanismes dérivationnels réguliers et, d'autre part, d'une créativité lexicale plus ou moins individuelle et disparate, qui s'éloigne des schémas traditionnels? Le rôle des paradigmes hybrides classiques est souligné par la plupart des auteurs (hybrides entièrement et partiellement confixaux, hybrides à préfixe allogène). Par contre, certains linguistes considèrent les hybrides surtout comme un type d'emprunt spontané dû, dans une large mesure, à des mécanismes plus ou moins subjectifs et expressifs de la créativité lexicale. Tel est l'avis de Hagège (1983), par exemple, qui étudie le rôle d'éléments redondants dans la création de certains hybrides. Ainsi, en nahuatl, il existe un hybride à fonction grammaticale loke- $\lambda$ en (/ce que/), composé d'un élément espagnol, lo que (/ce que/) et d'une base indigène $\lambda e n$, qui a le même sens (1983: 57). Cette opinion est partagée par Buttler (1986, 1990), selon laquelle les hybrides lexicaux relèvent surtout de la néologie d'auteur. 
À notre connaissance, limiter l'hybride lexical à des schémas dérivationnels tout récents, non assimilés, souvent à caractère subjectif, occasionnel et individuel, comme le veulent Hagège et Buttler, c'est trop restreindre le concept en question; et ce, en dépit de beaucoup d'auteurs qui y incluent également, ce que nous avons déjà montré, des modèles paradigmatiques réguliers et productifs, dont certains jouissent d'une longue tradition dans la langue. Tout compte fait, le degré de régularité et de productivité paradigmatiques n'est pas pour nous un critère pertinent permettant de délimiter le concept en question. L'hybride lexical peut englober autant les formations régulières et traditionnelles que des productions néologiques récentes se rapprochant parfois de l'hapax.

Voyons, à présent, une autre question cruciale qui divise les lexicologues, notamment les slaves: peut-on considérer comme des hybrides lexicaux seulement les formations dont le schéma est "base indigène + affixe étranger», ou bien faut-il également inclure les lexies à schéma dérivationnel inverse, soit «base allogène + affixe indigène»?

Certains linguistes francophones (Guiraud 1968; Humbley 1974; Trescases 1983) optent pour la dernière solution. Cependant, nous sommes d'avis que les dérivés à suffixe indigène, comme kitchenette, mixer, tester, flirter, quel que soit le statut de leurs bases lexicales (empruntées soit directement à une langue étrangère, comme kitchen-, mix-, soit indirectement, comme test-, flirt-), ne peuvent pas être qualifiés d'hybrides, mais plutôt d'emprunts assimilés au niveau dérivationnel. Il est évident que ce type d'emprunt présente, lui aussi, un certain degré d'hybridité, vu sa structure étymologiquement contrastée, où alternent de manière plus ou moins nette des éléments étrangers et indigènes. Néanmoins, un amalgame de traits allogènes et indigènes, inhérent à tout emprunt intégré dans un nouveau système, s'observe non seulement sur le plan dérivationnel, mais aussi au niveau phonique (par exemple, la prononciation «franglaise» de l'emprunt top secret), graphique (fr. bouledogue / ang. bulldog), flexionnel (par exemple, la possibilité de former le pluriel «à la française» pour les anglicismes du type barmans), etc. Il ne faut donc pas confondre l'hybridité des emprunts et celle qui caractérise les néologismes créés au moyen de formants allogènes. Dans le premier cas, l'hybridité, observable à tous les niveaux de la langue, découle de l'adaptation d'un emprunt aux mécanismes du système indigène. C'est au plan dérivationnel que cette hybridité est la plus visible, d'où une tendance de certains linguistes à appeler de tels emprunts hybrides. Dans le deuxième cas, l'hybridité est surtout liée au mécanisme générateur permettant de créer de nouvelles lexies au moyen de différents types morphologiques de formants allogènes (confixes: néo-classique, affixes: couill-av, mots pleins liés: feld-maréchal) qui se combinent soit avec une base indigène (secondairement, étrangère assimilée), soit avec un élément venant d'une autre langue étrangère (somno-+-gène). En résumé, dans le cas des vrais hybrides, l'opposition: indigène / allogène (allogène $\mathrm{A}$ / allogène $\mathrm{B}$ ) est un trait inhérent, en premier lieu, au mécanisme dérivationnel qui génère une lexie à formant(s) étranger(s), alors que dans le cas des emprunts, l'hybridité peut ne pas affecter le mécanisme dérivationnel. Si elle s'observe sur ce plan, une formation affixale à base étrangère a un formant indigène. Le mécanisme d'hybridation est donc contraire à celui de l'assimilation des emprunts au niveau dérivationnel, ce dernier aboutissant à l'allogénéité décroissante d'une lexie générée par rapport à sa base lexicale d'origine étrangère. Les deux mécanismes dérivationnels opposés peuvent être illustrés par le schéma suivant: 
TABLeAu 1

Mécanismes dérivationnels de l'emprunt (assimilé) et de l'hybride

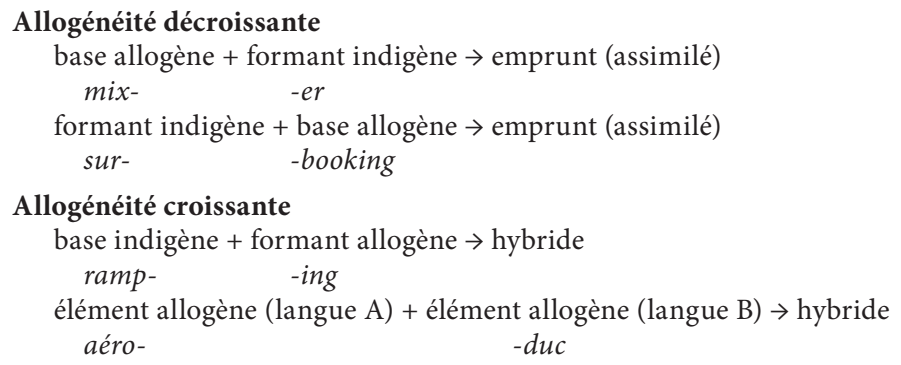

L'hybridation serait, dans cette optique, un processus de créativité lexicale qui combine les mécanismes de dérivation et d'emprunt. Ce processus aboutit à l'allogénéité croissante d'un dérivé par rapport à sa base lexicale, ce qui est contraire au mécanisme d'assimilation dérivationnelle de l'emprunt lexical, qui fonctionne en sens inverse (l'allogénéité d'un dérivé par rapport à sa base lexicale est décroissante). L'hybridation, comme nous la concevons, n'opère donc au niveau de l'affixation que selon le schéma suivant:

TABLEAU 2

Hybridation se situant au niveau de l'affixation

- base indigène + formant allogène $\rightarrow$ hybride hypertension, bronzing

\subsubsection{Allogénéité d'un formant hybride}

Nous avons montré que c'est le caractère allogène du formant d'une unité générée qui doit conférer à une nouvelle lexie le statut d'hybride. Comme l'allogénéité d'un élément morphématique est un concept variable suivant les différents degrés d'assimilation de cet élément dans la langue emprunteuse, il n'est pas toujours facile d'en tracer les contours. En ce qui concerne la création des hybrides, il semble que l'un des critères d'allogénéité du formant soit la non-autonomie de celui-ci dans la langue emprunteuse, par opposition à l'autonomie de son équivalent dans la langue prêteuse (voir la section 4.1.1.). En d'autres termes, il s'agit d'un élément directement emprunté à une langue étrangère pour créer un dérivé hybride (Obara 1986). Ce critère semble confirmer son utilité pour délimiter surtout les hybrides par composition (feldmaréchal; Feld: un nom en allemand), par recomposition de type gréco-latin (néoclassique; neos: un adjectif en grec), ainsi que les hybrides préfixaux (subalpin; sub: une préposition en latin). Cette méthode permet aussi de distinguer les hybrides de structures formellement apparentées, comme certains recomposés modernes ou mots-valises. Ainsi, dans un hybride télévision, télé- est un emprunt direct au grec quant à la forme et au sens (/loin/, /de loin/), alors que téléspectateur est un recomposé moderne (le même élément étant issu d'une apocope de télévision). Interclasse est un hybride, car l'élément inter- est directement emprunté au latin, tandis que la formation internaute est un mot-valise né d'un télescopage de deux noms préexistant en français (internet + astronaute).

Bien que certains formants hybrides puissent avoir parfois une certaine autonomie en français, celle-ci n'est pas de même nature que dans la langue d'origine 
(ex ou super, prépositions en latin, ne jouent aucunement un rôle analogue en français). Cette autonomie est le résultat d'une conversion à partir d'un hybride de base: $d u$ supercarburant $\rightarrow d u$ super. L'autonomie d'un élément gréco-latin en français peut également résulter d'un autre type de conversion, c'est-à-dire d'une adjectivisation (ce garçon est super). Le mécanisme analogue d'autonomie secondaire des formants hybrides s'observe aussi dans le cas de certains confixes (une automobile $\rightarrow$ une auto, une télévision $\rightarrow$ une télé).

L'allogénéité d'un formant hybride de type préfixal peut être parfois accentuée par la présence en français de son équivalent autonome né par une voie évolutive régulière. Ainsi, il existe des doublets du type ultra- / outre(-), super- (supra-) / sur(-), etc. (où les deux membres de chaque paire ont un étymon commun) ou du type post/ après, sub- (infra-) / sous(-), etc. (où chacun des membres a une origine différente). Dans les deux cas, le premier élément d'un doublet, privé d'autonomie en français, est susceptible de conférer au dérivé qu'il forme avec une base lexicale un statut d'hybride. Ainsi, subalpin, infrarouge seront des préfixés hybrides, contrairement à sous-entendu; de même: superpuissance, supranational (mais surestime), ultra-violet (mais outre-mer), etc.

C'est la délimitation des hybrides suffixaux qui pose le plus de problèmes, l'allogénéité des suffixes étant moins nette que celle des autres types de formants. Si pour les formants hybrides non suffixaux un paramètre pertinent d'allogénéité peut être leur non-autonomie en français, contrairement à l'autonomie de leurs équivalents dans la langue prêteuse, ce critère ne s'applique pas aux suffixes, ceux-ci étant par nature des morphèmes liés.

Cette différence, permettant de comparer le degré d'allogénéité des différents types d'unités morphématiques, est illustrée par le schéma suivant:

TABleau 3

Degré d'allogénéité des différents types d'unités morphématiques

Langue prêteuse

- autonomie structura

- autonomie

Feld

mamma

huper

- non-autonomie -ista

\author{
Langue emprunteuse (français) \\ autonomie \\ structure (mot plein non lié) \\ non-autonomie \\ feld- (mot plein lié) \\ mammo- (confixe) \\ hyper- (préfixe) \\ non-autonomie \\ -iste (suffixe)
}

L'allogénéité des formants non suffixaux est particulièrement nette grâce au mécanisme de conversion qui caractérise le processus de leur emprunt: l'autonomie d'un élément dans la langue prêteuse s'oppose à la non-autonomie de cet élément dans la langue emprunteuse (feld-, mammo-, hyper-). En revanche, si les éléments étrangers conservent leur autonomie dans la langue d'arrivée, ils s'assimilent au système lexical de cette langue au point d'acquérir un statut d'unités indigènes (structure). Cette conversion n'a pas lieu lorsqu'on emprunte des éléments suffixaux, donc l'allogénéité de leurs équivalents dans la langue d'arrivée est moins nette (-iste). Par conséquent, étant une condition de l'hybridité, l'opposition base indigène / formant allogène se neutralise. L'application de l'opposition suffixale: savant / non savant (-aire / [-i]er 
de -arius, -al / -el de -alis, -ation / -aison de -atio, -ose / -eux de -osus, etc.), à l'instar de super- / sur- (voir plus haut) pour établir l'opposition correspondante: (formation suffixale hybride [avec formant savant] / formation suffixale non hybride [avec formant non savant]) s'avère peu opérante pour plusieurs raisons. D’abord, les suffixes savants se combinent très souvent avec des bases savantes non autonomes (insul-aire, marit-al), ce qui exclut automatiquement le statut hybride de telles formations. Ensuite, les inventaires de suffixes savants et non savants, dressés traditionnellement selon des critères phoniques et distributionnels, peuvent varier selon l'auteur. Certains linguistes, Marchand (1969) par exemple (après Zwanenburg 1983), distinguent même des suffixes neutres à l'égard du trait [ \pm savant], comme -able, -ance, -ant. La question reste toujours épineuse si un suffixe savant n'a pas son équivalent non savant. Des formations à base autonome savante, comme culturiste, peuvent-elles être considérées comme des hybrides?

Cependant, il existe des cas où s'observe un certain degré d'hybridité de formations suffixales. D’abord, il importe de distinguer l'allogénéité des suffixes non latins. Des structures comportant une base indigène à laquelle se joint -ing, suffixe emprunté à l'anglais (ramping), ou - $\boldsymbol{a} v$, suffixe tzigane (couillav), ont un caractère hybride bien marqué. Pour délimiter un statut plus ou moins hybride de formations à suffixe savant d'origine latine, il semble que l'on puisse proposer la solution de Buttler $(1986,1990)$, qui attribue le statut d'hybride aux suffixés polonais dont la structure morphématique est atypique ou néologique, trait qui met en relief l'allogénéité du suffixe par rapport à la base indigène. En français, une telle hybridité semble caractériser un schéma dérivationnel à productivité minime: base non savante + suffixe savant (dégoûtation, face à la formation régulière contenant le même suffixe accolé à l'allomorphe savant: dégustation). Ce modèle dérivationnel atypique est souligné entre autres par Corbin (1987: 91).

En nous appuyant sur les critères ci-dessus, nous pouvons proposer une définition de l'hybride lexical (celle-ci s'applique notamment au type structurel d'hybride décidément le plus répandu, à savoir l'hybride à deux éléments):

L'hybride lexical est un type néologique issu en premier lieu d'une hybridation:

- considérée comme un processus spécifique de créativité lexicale qui combine les mécanismes de dérivation et d'emprunt

- se réalisant par l'adjonction d'un élément allogène (mot plein lié, confixe ou affixe) venant directement d'une langue $A$

- soit à une base indigène (feld-maréchal, chocolatophilie, hypermarché)

- soit à un élément allogène venant directement d'une langue B (aéroduc).

Le français emprunte aussi des hybrides à d'autres langues où ils sont formés en vertu de mécanismes hybridogènes analogues (monocle, du bas latin monoculus). Dans ce dernier cas, l'emprunt conserve son statut d'hybride dans la langue d'arrivée à condition que sa structure hybride y soit perceptible synchroniquement. Les formants hybrides n'ont pas d'autonomie dans la langue emprunteuse.

\section{Typologie des hybrides lexicaux}

Nous ne la présentons qu'en grandes lignes, en proposant plusieurs classifications faisant appel à des critères différents. 


\subsection{Typologie morphologique}

C'est le classement principal des hybrides lexicaux qui se fait selon les différents types d'agencements morphématiques.

\subsubsection{Hybrides par composition}

La composition, conçue au sens large du mot, comprendra tant les formations traditionnelles que les synapsies et les lexies complexes. Nous y intégrons aussi des sigles et des mots-valises. La classification ci-dessous n'englobe que les types les plus réguliers, en passant sous silence des variantes nominales composites ou fantaisistes (démmerdenzisich).

- nom + nom: gestalt-thérapie, médecine-ball

- nom + adjectif: french cancan, Clean-émail

- nom + autre partie du discours: Café Ludo, égo-training

- adjectif + adjectif: sanctosépulcral, austro-prussien

- synapsie: harpon de spudding, toiture à sheds

- lexie nominale complexe: light emitting diode, amplificateur de public address

- mots-valises: Velourex (velours + rex), Incoloy (incolore + alloy)

- sigles: F.I.V. (fécondation in vitro), D.N.S. ("Domaine Name Server»)

\subsubsection{Hybrides par confixation}

Parmi tous les types morphologiques d'hybrides, ce schéma classique (notamment la confixation partielle) est décidément le plus productif. On peut distinguer des hybrides entièrement confixaux (gréco-latins), partiellement confixaux (base indigène combinée à un confixe antéposé ou postposé), ainsi que toutes sortes d'expansions confixales.

- confixation totale: lactogène, aéroduc

- confixation partielle: néoclassique, cancérologie

- expansions: hypofifriophobie, fruidemérologue

\subsubsection{Hybrides par affixation}

Par rapport au type précédent, ils présentent moins de formants hybrides et une productivité moins élevée.

- préfixés hybrides: hypercorrection, infrarouge

- suffixés hybrides: bronzing, tirav

\subsubsection{Hybrides locutionnels}

Caractéristiques surtout du français familier et branché, ils se divisent en deux soustypes principaux:

- locutions verbales: être croque-love, être low-tech

- locutions adverbiales: grosso merdo, what mille de temps

\subsection{Hybrides par dérivation et hybrides par emprunt}

Les hybrides peuvent être soit formés en français, soit empruntés à d'autres langues. Les hybrides par dérivation, beaucoup plus nombreux que ceux par emprunt, se 
forment à la suite du processus d'hybridation que nous avons étudié plus haut. En ce qui concerne la deuxième catégorie d'hybrides, deux cas sont à distinguer. Premièrement, un hybride peut être créé dans une langue autre que le français. Celui-ci ne fait que l'emprunter, en l'assimilant à plusieurs niveaux, notamment grapho-phonique. Ainsi, le nom monocle n'est pas un hybride par dérivation en français contemporain, mais un hybride-emprunt né en bas latin: mono $[\underline{s}]$, formant grec + oculus, base latine. Le mot automobile n'aura un statut d'hybride par dérivation que dans la langue où il a été formé (français), alors que dans les langues qui l'ont emprunté, il sera tout naturellement un hybride-emprunt (automobil en polonais, par exemple).

Un mécanisme en quelque sorte inverse s'observe dans certains hybrides par emprunt, comme ventôse. Comme le fait observer Leclercq (1989: 310), c'est un mot emprunté au latin de forme adaptée. Son étymon (ventosus) n'a pas de statut hybride. L'hybridité n'apparaît qu'en français, sous la Révolution où ce nom a été emprunté au latin, et résulte d'un contraste qui s'est produit entre une base lexicale de type indigène et un formant allogène. L'allogénéité de -ôse se fait voir par rapport à son équivalent populaire -eux dans venteux. En revanche, nivôse n'est évidemment pas un hybride, car ses deux éléments morphématiques sont de type savant, par opposition à leurs équivalents non savants dans neigeux.

\subsection{Hybrides interférentiels et non interférentiels}

Certains hybrides sont dus aux interférences de deux ou de plusieurs langues qui sont en concurrence à la suite d'un bilinguisme établi dans des zones à population mixte ou dans des territoires limitrophes. Nous proposons de les appeler hybrides interférentiels, par opposition aux hybrides non interférentiels.

Les hybrides non interférentiels correspondent grosso modo à ce qu'on appelle traditionnellement hybrides dénominatifs, alors que les hybrides interférentiels englobent un grand nombre de formations onomastiques. Les hybrides interférentiels de type onomastique (et tout particulièrement toponymiques) paraissent plus ou moins stables, dans la mesure où ils donnent naissance par exemple à des noms géographiques (ainsi, le bilinguisme anglo-français en Louisiane aux XVIII ${ }^{\mathrm{e}}$-XIX ${ }^{\mathrm{e}}$ siècles a fait naître bon nombre de structures hybrides toponymiques, contenant le formant français -ville, ajouté à des noms anglais, comme Greenville, Nashville, Hopkinsville, Huntsville, Kirksville, etc.; Rudnyćkyj 1972: 300); par contre, les hybrides interférentiels de type dénominatif sont souvent des néologismes occasionnels et peuvent soulever des objections normatives. Voyons, pour illustrer ce dernier cas, un hybride préfixal disconnecté (au lieu de déconnecté), créé par les ressortissants français expatriés à Toronto, une ville anglophone (Forlot 1999: 122).

Comme notre travail porte essentiellement sur les hybrides non interférentiels, nous ne présentons ci-dessous que quelques observations d'ordre méthodologique concernant les hybrides interférentiels.

Les linguistes francophones étudient les hybrides onomastiques surtout du point de vue diachronique (voir surtout Nègre 1990; Dorion et Poirier 1975; Hamon 1992). Ainsi, Nègre (1990) divise les formations hybrides toponymiques en dérivés hybrides (ex.: Bugnières: germ. Bunno + suffixe roman fém. pl. - $\underline{\text { arias }}$; Bunn-arias [terras] : /terres de Bunno/) et composés hybrides (ex. : Basemont: germ. Baso + lat. montem). L'étude des hybrides interférentiels, telle qu'elle est faite traditionnellement, n'est donc 
pas compatible avec notre théorie. D’abord, elle est essentiellement diachronique, notamment pour ce qui est des hybrides onomastiques, et ensuite, cette approche confond les hybrides et les emprunts assimilés au niveau dérivationnel.

\subsection{Hybrides dénominatifs et hybrides d'auteur}

Suivant la fonction du néologisme, on peut diviser les hybrides en deux types principaux: hybrides dénominatifs et hybrides d'auteur.

\subsubsection{Hybrides dénominatifs}

C'est la première fonction des hybrides lexicaux. Créés pour dénommer des concepts nouveaux utilisés surtout dans les différents domaines techniques et scientifiques, ces hybrides empruntent leurs formants allogènes notamment au latin et au grec, langues qui, depuis le XviII ${ }^{\mathrm{e}}$ siècle, prêtent leurs ressources lexicales et ne cessent de le faire aujourd'hui. Généralement, il s'agit là des internationalismes hybrides, formés de manière à avoir trois qualités essentielles: a) outil de communication international; b) économie et concision formelles assurées surtout par leur structure entièrement ou partiellement confixale; c) précision sémantique (cet aspect est souligné entre autres par Buttler 1990 et Corbin 1987). Pour illustrer le dernier point, mentionnons l'opinion de Corbin (1987), qui met en relief la spécialisation sémantique (notamment la fonction classifiante) de certains néologismes à formant savant («archéoconstituant», pour citer l'auteur), soit préfixal, soit confixal. C'est ce formant qui fait fonction de classificateur, en spécifiant les différentes caractéristiques de localisation spatiale ou temporelle (transatlantique, exocentrique, intracrânien...), la quantification (monosite, bipolaire, microampère, nanoseconde...), etc.

\subsubsection{Hybrides d'auteur}

Observables surtout dans le style littéraire et différents sociolectes (langages familier, argotique ou branché), ils assument des fonctions poétiques, satiriques, expressives, ludiques, etc. Le mécanisme de leur formation consiste généralement à combiner des éléments contrastés stylistiquement ou sémantiquement (pluricon, trouillomètre, toilettologue), éléments qui, normalement, devraient être séparés (Marchand 1969; Asher 1984; Cellard et Rey 1991; Szymanek 1989; Buttler 1986, 1990)

Comme nous l'avons déjà vu, pour certains auteurs (Beciri 1994; Cabré1998) cette hybridité de registre, de style ou de sémantisme est le principal critère permettant de conférer aux unités lexicales un statut d'hybride. Ainsi, l'hétérogénéité étymologique (notamment pour les formations savantes), paramètre traditionnel de l'hybridité lexicale, n'est pas selon eux un critère pertinent, de telles formations n'ayant rien d'irrégulier ou d'hybride. Par conséquent, un hybride traditionnel comme spectroscope n'est, selon ces linguistes, qu'un composé savant non hybride, car les deux éléments de ce mot, bien que divergents étymologiquement, ne sont caractérisés par aucun contraste de registre, de style ou de sémantisme.

\section{Conclusion}

En fonction de la théorie selon laquelle l'hybridation repose sur un mécanisme dérivationnel d'adjonction de différents types de formants directement empruntés à une 
ou plusieurs langues étrangères, l'hybride lexical est un type néologique spécifique qui unit les traits de dérivé et d'emprunt. Cette approche permet de distinguer ce concept des notions apparentées (surtout des emprunts assimilés dérivationnellement), en démontrant que l'hybride et l'emprunt assimilé sont deux types de créations lexicales basées sur des mécanismes opposés, et, par conséquent, ils ne devraient pas être confondus. En même temps, elle élargit la notion d'hybride en dehors de son acception classique (surtout confixale), qui est la seule à avoir jusque-là des cadres méthodologiques plus ou moins stricts.

L'hybride lexical est un concept scalaire dont les contours catégoriels sont parfois flous, surtout en ce qui concerne les hybrides suffixaux à base française et formant «latinisant». La néologie hybride, comme on l'a vu plus haut, revêt de nombreux schémas morphématiques. Outre les hybrides classiques d'origine gréco-latine, on crée aujourd'hui des néologismes hybrides surtout au moyen d'éléments anglo-américains (docu-soap, top-niveau), sporadiquement à l'aide d'éléments venant d'autres langues, ce qui n'empêche pas la confixation gréco-latine d'être toujours en expansion, notamment dans les langages scientifiques et techniques. Il semble d'ailleurs que parmi les différents types structurels d'hybrides, l'hybridation confixale demeure la plus productive. Quérin (2001), par exemple, a répertorié 249 hybrides gréco-latins en français médical. C'est en premier lieu la confixation partielle qui jouit d'une grande productivité aujourd'hui, type caractéristique surtout du langage des médias et de la publicité. Bon nombre de ces formations sont des hybrides d'auteur à caractère occasionnel et structure plus ou moins composite (Sablayrolles 2000). Deux sous-types structurels se distinguent ici: les éléments confixaux peuvent se joindre soit aux bases indigènes autonomes (aéroville, multimodal, médiacratie, toilettologue, sartrophage, chocolatophilie), soit aux bases indigènes tronquées (publiphobe, télévore, europhile, écocide). Dans les hybrides fantaisistes, créés surtout dans les buts publicitaires, les frontières entre confixation et composition sont souvent diluées, la structure composite d'un hybride évoquant le caractère exotique et nouveau d'un référent. Tel est le cas d'un néologisme publicitaire lipo-action (marque de lessive), où le premier élément, par son trait de nouveauté lexicale (du grec lipos, /graisse/), semble perdre son statut de confixe, du moins pour le locuteur moyen. Un autre exemple de cet «exotisme structurel» est un hybride Topkapil (nom propre d'un produit de beauté), morphologiquement identifiable à un mot-valise (Topkapi: nom du fameux palais de sultan à Istanbul, comme symbole de luxe + capil: allomorphe fantaisiste latinisant de capillus, /cheveu/ en latin), et qui, connotativement, a un formant hybride de plus (top, élément anglais évoquant l'idée de supériorité).

Parmi les hybrides par affixation, ceux qui sont les plus productifs ont une structure préfixale (subaigu, ultra-chic, anticorps, hypercorrect, etc.). Parmi les formants non classiques, l'affixe hybride qui semble le plus productif est le suffixe anglais -ing qu'on accole à des bases françaises (ramping, bronzing, frotting). Un autre formant non classique est - $\boldsymbol{a} \boldsymbol{v}$, la désinence infinitive d'origine tzigane ajoutée aux bases françaises, qui fait fonction de suffixe dans le français des cités (pourav, couillav, tirav). D’autres affixes allogènes non classiques s'observent sporadiquement (overvitaminé, ordurissimo).

\section{NOTES}

1. Les exemples sont empruntés à Forlot (1999).

2. La plupart des exemples de ce classement sont empruntés à Sablayrolles (2000). 


\section{RÉFÉRENCES}

Asher, Ronald E., dir. (1984): The Encyclopedia of Language and Linguistics. Oxford: Pergamon Press.

BeCIRI, Hélène (1994): Néologismes «spécialisés » dans les médias et la vie quotidienne: aspects morphologiques et sémantiques. Néologie lexicale. 6:302-354.

Benveniste, Emile (1967): Fondements syntaxiques de la composition nominale. Bulletin de la Société de Linguistique de Paris. 62(1):15-31.

Berrendonner, Alain (1997): Pléonasmes syntaxiques: dédoublement ou hybridation? Langue française. 115:75-87.

ButtLer, Danuta (1986): Formacje hybrydalne w różnych okresach rozwoju i warstwach słownikowych polszczyzny. In: Stefan Warcho£, dir. Formacje hybrydalne w językach słowiańskich. Lublin: UMCS, 24-31.

Buttler, Danuta (1990): Hybrydy w nowym słownictwie polskim. Poradnik Językowy. 2:145-150.

CABré, Maria-Teresa (1998): La terminologie; théorie, méthode et application. Ottawa: Presses de l'Université d'Ottawa.

Cellard, Jacques (1998): Les racines grecques du vocabulaire français. Bruxelles: Duculot.

Cellard, Jacques (2000): Les racines latines du vocabulaire français. Bruxelles: Duculot.

Cellard, Jacques et Rey, Alain (1991): Dictionnaire du français non conventionnel. Paris: Hachette.

Chaurand, Jacques (1977): Introduction à l'histoire du vocabulaire français. Paris: Bordas.

Corbin, Danielle (1987): Morphologie dérivationnelle et structuration du lexique. Tübingen: Niemeyer.

Corbin, Danielle (1991): La formation des mots: structures et interprétations. Lexique. 10:7-30.

DARMESTETER, Arsène (1926): Cours de grammaire historique de la langue française (Formation des mots et vie des mots). Paris: Librairie Delagrave.

Darmesteter, Arsène et HatzFeld, Adolphe (1964): Dictionnaire général de la langue française du commencement du XVII siècle jusqu'à nos jours. Vol. 1. Paris: Delagrave.

Dauzat, Albert, Dubois, Jean, Mitterand, Henri, et al. (1964): Nouveau dictionnaire étymologique et historique. Paris: Larousse.

DePeCKer, Loïc (2000): Quelques aspects des vocabulaires spécialisés de la fin du $\mathrm{xx}^{\mathrm{e}}$ siècle: entre créativité lexicale et parallélisation sur l'anglais. La banque des mots. 60:103-134.

Dorion, Henri et Poirier, Jean (1975): Lexique des termes utiles à l'étude des noms de lieux. Québec: Presses de l'Université Laval.

Dubois, Jean, Giacomo, Mathée, Guespin, Louis, et al. (1973): Dictionnaire de linguistique. Paris: Larousse.

Duвоis, Jean (1999): La dérivation suffixale en français. Paris: Nathan.

ForLot, Gilles (1999): Variation lexicale et et reconstruction sémantique en migration: le cas de Français expatriés en milieu anglo-canadien. La linguistique. 2(35):113-126.

Galliot, Marcel (1954): Essai sur la langue de la réclame contemporaine. Toulouse: E. Privat.

Gaudin, François et Guespin, Louis (2000): Initiation à la lexicologie française (De la néologie aux dictionnaires). Bruxelles: Duculot.

GoudaIllier, Jean-Pierre (1997): Comment tu tchatches! Dictionnaire du français contemporain des cités. Paris: Maisonneuve et Larose.

Grevisse, Maurice (1975): Le Bon Usage. Gembloux: Duculot.

Gross, Gaston (1996): Les expressions figées en français. Paris: Ophrys.

Guilbert, Louis (1975): La créativité lexicale. Paris: Larousse.

Guiraud, Pierre (1968): Les mots savants. Paris: PUF.

HAGÈGe, Claude (1983): Voies et destins de l'action humaine sur les langues. In: Istvan Fodor, dir. La réforme des langues. Vol. 1. Hamburg: Buske, 11-68.

Hamers, Josianne et Blanc, Michel (1983): Bilingualité et biliguisme. Bruxelles: Mardaga.

Hamon, Albert (1992): Les mots du français. Paris: Hachette. 
Huber, Herbert et Cheval, Mireille (1996): Glossaire: noms composés par juxtaposition. Lebende Sprachen. 41:179-181.

Humbley, John (1974): Vers une typologie de l'emprunt linguistique. Cahiers de lexicologie. 25(2):46-70.

Ǩ̌ EČKová, Vlasta (2000): Tvorenie pomenovaní v súčasnej francúzštine. Banská Bystrica: Fakulta humanitných vied, UMB.

LeClerCQ, Henri (1976)): À propos des néologismes d'origine grecque. Les études classiques. 44(2):97-108.

LeClerCQ, Henri (1989): Néologismes d'origine grecque et latine pendant la Révolution française. Les études classiques, 57(4):303-316.

Marchand, Hans (1969): The Categories and Types of Present-Day English Word-Formation. München: Verlag C. H. Beck.

Mathieu-Colas, Michel (1996): Essai de typologie des noms composés français. Cahiers de lexicologie. 69(2):71-125.

Merle, Pierre (1999): Le dico du français branché. Paris: Seuil.

NÈGRE, Ernest (1990): Toponymie générale de la France. Genève: Libraire Droz.

Nicolas, Christian (1994): Le procédé du calque sémantique. Cahiers de lexicologie. 65(2):75-101.

Ова RА, Jerzy (1986): Hybrydy i półkalki na tle innych jednostek językowych obcego i rodzimego pochodzenia. In: Stefan WARCHO£, dir. Formacje hybrydalne w językach słowiańskich. Lublin: UMCS, 59-73.

Quérin, Serge (2001): Emploi de termes hybrides gréco-latins dans le langage médical. Meta. 46(1):7-15.

Rey-Debove, Josette (1984): Le domaine de la morphologie lexicale. Cahiers de lexicologie. 45(2):3-19.

Rheims, Maurice (1969): Dictionnaire des mots sauvages. Paris: Larousse.

Robert, Paul (1992): Le grand Robert de la langue française. Dictionnaire alphabétique et analogique de la langue française. Paris: Le Robert.

RudNYĆKYJ, Jaroslav Bohdan (1972): Names in contact: Canadian pattern. In: Henri Dorion, dir. Les noms de lieux et le contact des langues. Place Names and Language Contact. Québec: Presses de l’Université Laval, 293-301.

Sablay rolles, Jean-François (2000): La néologie en français contemporain. Paris: Honoré Champion.

Spence, Nicol (1991): Les mots français en -ING. Le français moderne. 59(2):188-213.

Sy PNICKI, Józef (1979): La composition nominale en français et en polonais. Poznań: Wydawnictwo Naukowe UAM.

Szymanek, Bogdan (1989): Introduction to Morphological Analysis. Warszawa: PWN.

Thiele, Johannes (1987): La formation des mots en français moderne. Montréal: Les Presses de l'Université de Montréal.

Trescases, Pierre (1983): Aspects du mouvement d'emprunt à l'anglais reflétés par trois dictionnaires de néologismes. Cahiers de lexicologie. 42(2):86-101.

VaUgelas, Claude Favre (1981): Remarques sur la langue française. Paris: Champ Libre.

WARChO£, Stefan (1986): O derywacji hybrydalnej. In: Stefan Warcho£, dir. Formacje hybrydalne w językach słowianskich. Lublin: UMCS, 107-122.

Zwanenburg, Wiecher (1983): Productivité morphologique de l'emprunt: étude des dérivés déverbaux savants en français moderne. Amsterdam: John Benjamins. 